\title{
La vengeance clanique dans les contes épiques
}

\section{nanaïs}

Essai d'interprétation à partir de conceptions chamaniques

Clanic Revenge in Nanai Epic Tales: An interpretation from a shamanistic point of view

\section{Tatiana Bulgakova}

\section{(2) OpenEdition}

\section{Journals}

Édition électronique

URL : https://journals.openedition.org/emscat/1304

DOI : 10.4000/emscat.1304

ISSN : 2101-0013

Éditeur

Centre d'Etudes Mongoles \& Sibériennes / École Pratique des Hautes Études

Édition imprimée

Date de publication : 1 décembre 2001

Pagination : 163-172

ISBN : 2-9518888-0-5

ISSN : 0766-5075

Référence électronique

Tatiana Bulgakova, «La vengeance clanique dans les contes épiques nanaïs », Études mongoles et sibériennes, centrasiatiques et tibétaines [En ligne], 32 | 2001, mis en ligne le 17 mars 2009, consulté le 13 juillet 2021. URL : http://journals.openedition.org/emscat/1304; DOI : https://doi.org/10.4000/ emscat.1304

Ce document a été généré automatiquement le 13 juillet 2021.

(c) Tous droits réservés 


\title{
La vengeance clanique dans les contes épiques nanaïs
}

\author{
Essai d'interprétation à partir de conceptions chamaniques \\ Clanic Revenge in Nanai Epic Tales: An interpretation from a shamanistic point \\ of view
}

Tatiana Bulgakova

\section{NOTE DE L'ÉDITEUR}

Traduit et adapté par Marie-Lise Beffa et Laurence Delaby.

1 Le thème de la vengeance clanique se rencontre dans les contes nanaïs. Son étude d'après les matériaux issus du genre appelé niyman peut contribuer à éclairer les nombreuses questions que l'on se pose sur la naissance du folklore ${ }^{1}$.

2 Il est couramment admis que les contes sont des fictions. On convient néanmoins que leur contenu prend sa source «dans une réalité historique passée» (Propp 1986, p. 353). Les contes se seraient petit à petit éloignés de cette réalité pour s'orienter vers la fiction à une étape ultérieure de leur constitution. Le nimman, lui, ne s'en serait pas tellement éloigné, puisque, selon les chamanes nanaïs, ce serait un récit d'événements réels qui se seraient produits dans le passé et qui se produiraient encore à présent, comme eux-mêmes ainsi que des gens de leur connaissance peuvent en témoigner. Nous avons eu l'occasion, au cours de nos recherches, d'interroger des personnes qui assurent avoir participé à de tels événements, et il nous a ainsi été donné de considérer le nizman à la lumière des représentations des porteurs de la tradition.

3 Le thème de la vengeance clanique est plus caractéristique des épopées que des contes. C'est pourquoi Ju. A. Sem (1986, p. 41) a proposé d'appeler «contes épiques» les niyman dont il avait eu connaissance pour avoir assisté à leur récitation. Traditionnellement, un niyman commence par une série de vers allitérés qui parlent de la solitude du héros dans son campement - solitude qui est récente. 


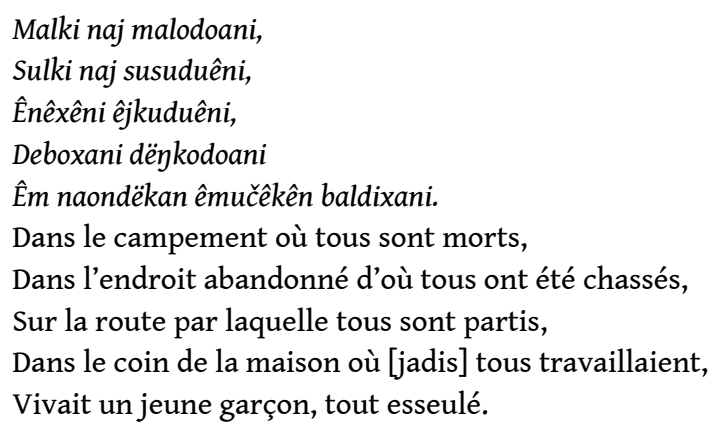

4 Notre héros grandit sous la surveillance de protecteurs invisibles, qui un jour lui apparaissent et l'envoient «accomplir le devoir sacré de vengeance contre l'ennemi qui a attaqué son campement, emmené en captivité ses parents, assassiné les membres de son clan» (Sem 1986, p. 41). L'apparence de ces protecteurs est insolite. «Du rivage monte un petit vieux. Oh la la! Il est large d'une brasse, haut d'une brasse! Ses jambes s'enfoncent dans le sol jusqu'au genou.» ${ }^{2}$ Le héros grandit, devient un mêrgên [un preux]. Il part à la recherche de l'ennemi qui a tué son père, le provoque en duel et le vainc. Les détails du combat ne sont guère vraisemblables. Au cours de la lutte, le héros lève les yeux : «Un oiseau plane. L'oiseau dit au jeune preux : "Arrête-toi un instant ! Je t'envoie une boule d'argent, une boule d'or. Ne les laisse pas tomber à terre !..." Le preux s'en saisit, les ouvre. Dedans se trouve le putê [l'âme chamanique de Gagdanču, l'ennemi du héros]. Il tire le putê par une jambe - et la jambe de Gagdanču s'envole. Il se saisit d'un bras [du putê] et le tire - et le bras de Gagdanču s'envole»³ (Sunik 1958, p. 120).

Certes, la vengeance de sang a autrefois existé chez les Nanaïs (Lopatin 1922, p. 187). Mais ce n'est pas cette réalité ethnographique qui est à chercher dans le conte cité plus haut : la vengeance de sang qui y est décrite n'est pas celle que les Nanaïs pratiquaient, mais celle, fantasmatique, qu'ils voyaient en rêve et lors des visions chamaniques ${ }^{4}$. La parenté entre nigman nanaï et séance chamanique se trouve confirmée par la similitude de leurs chants récitatifs ${ }^{5}$, par le fait que leur exécution a lieu dans les mêmes conditions de nuit et d'obscurité et qu'elle s'adresse avant tout aux esprits et non aux humains. Enfin, le ninman et la séance chamanique sont désignés par des termes dérivant d'une même racine : nigmanbori «raconter un conte», nigmačiori «chamaniser». Par ailleurs, le conte nanaï n'est pas seulement proche de la pratique chamanique, il en est une manifestation. Et tant que le chamanisme restera vivace dans la région de l'Amour, l'apparition de nouveaux contes sera toujours possible: c'est du moins ce qu'affirment les chamanes nanaïs.

Quelle pourrait en être la raison? La nature des relations du chamane avec les esprits représente un savoir ésotérique inaccessible à la collectivité et l'on croit que les esprits punissent de mort le chamane qui le divulguerait. Il arrive néanmoins que, dans de rares cas - en particulier si une mort rapide parait inévitable au chamane, ou bien désirable pour une raison quelconque -, le chamane se décide à raconter aux gens quelque chose de sa vie secrète dans le monde des esprits ${ }^{6}$. Comme il craint la réprobation de ses auditeurs, il narre ses aventures de façon détournée, à savoir sous la forme d'un conte. Il fait comme si le récit ne parlait pas de lui mais d'un autre. Ceux qu'il appelle mêrgên [preux] et pudin [jouvencelles] sont en réalité lui-même, les autres chamanes et les divers esprits, de sorte que seul l'initié peut percer le secret du récit. 
Ainsi se fait le codage nécessaire de l'information lors de la transmission d'une connaissance ésotérique.

Nous nous sommes intéressée aux expériences vécues par ces chamanes qui maîtrisent le code des contes et nous avons tenté de comprendre l'origine et la signification du thème de la vengeance clanique. Pourquoi est-ce un héros solitaire - même s'il arrive parfois qu'il ait des frères - qui est appelé à venger des pères et des aïeux depuis longtemps trépassés? Selon les explications des chamanes, c'est que ce héros est un futur chamane, choisi par les esprits parmi les nombreux descendants d'une lignée donnée.

Notons que, chez les Nanaïs, la transmission du don chamanique se fait strictement selon une ligne clanique déterminée, paternelle de préférence (Smoljak 1991, p. 35). L'individu consacré chamane hérite donc les esprits chamaniques de sa lignée paternelle. Ces esprits sêvên, qu'en règle générale ni lui-même ni ses ancêtres n'ont jamais vus pour de vrai ${ }^{7}$, l'obligent à poursuivre la lutte séculaire. Il faut dire que l'hostilité entre chamanes puissants était considérée comme inévitable. Bien que les rivalités nées de questions de prestige et de querelles à propos des terrains de chasse aient été affaires courantes, ce ne sont point elles qui donnaient lieu à cette relation d'hostilité entre chamanes, mais les conflits censés provoqués par leurs sêvên.

- Se faire un ennemi, c'est très facile, se plaint Nikolaj Petrovič Bel'dy qui souffre d'une maladie chamanique.

- Même si l'on vit prudemment?

- Prudemment? Mais je vis prudemment ! C'est en rêve seulement que je ${ }^{8}$ me suis changé en oiseau et que j'ai becqueté le plancton de la berge. Soudain, j'ai picoré une bestiole. Brusquement, j'ai compris que j'avais gobé le panjan [l'âme] d'un gosse. Et vlan! Au matin, le bébé était mort. Voilà-t-il pas que son père, ou sa tante ou un des leurs, se trouve être un chamane plus puissant que moi. Ils vont chamaniser, et ils sauront que le responsable de la mort du petit, c'est moi. À tous coups, ils vont s'en prendre à moi! Et alors je vais être comme un ennemi de sang pour eux. Et pourtant, je ne suis pas coupable. ${ }^{9}$

Ainsi, les chamanes déplorent être cause de malheurs pour autrui sans le faire exprès : «C'est le sêvên qui fait le coup, explique la chamanesse Lindzja Bel'dy, il embrouille tout. Le chamane, lui, ne sait pas d'où le malheur est venu. Est-ce que vraiment un chamane ferait du mal à quelqu'un volontairement? C'est son sêvên qui se mêle de tout comme un chien mauvais.»

11 Lors de leurs combats, les chamanes ferment les routes de leurs adversaires dans le monde invisible, ils détruisent les abris où ces adversaires sont censés se réfugier et ceux où ils sont censés garder les âmes de leurs patients, ils emprisonnent les sêvên de ces adversaires.

Par exemple, si l'âme d'un chamane - voire sa vie - est menacée au cours de la lutte, il donnera à l'ennemi, à la place de la sienne, l'âme d'un de ses patients en cure ou celle d'un jeune de sa famille, qui en mourra. Par ailleurs, si un chamane finit par vaincre son ennemi, non seulement il le met à mort, mais il s'efforce d'exterminer toute sa parenté, les enfants en particulier (Smoljak 1991, p. 60). Et cela, pour priver son ennemi de successeurs susceptibles de poursuivre la lutte de génération en génération. Les esprits du clan exterminé, ne trouvant plus de nouveau maître du même sang, sont obligés de quitter le territoire du clan. Ainsi, le chamane vainqueur se croit à l'abri : il ne craint plus que grandisse un jour un héritier de l'ennemi vaincu qui de nouveau menacerait son bien-être et celui de ses enfants. 
13 C'est pourquoi, dans les contes que nous avons étudiés, l'action commence par ces mots : «En des lieux désertés par le père» ou encore «Absinthe et herbe..., personne, rien» (Sunik 1958, p. 114). Nous sommes au campement d'un chamane qui a péri et dont l'ennemi s'est appliqué à anéantir toute la parenté. En dépit de tous ses efforts, est resté en vie un petit garçon échappé à sa vue ${ }^{10}$. C'est le futur vengeur. Malgré son esseulement, il ne périra pas. Sa vie est préservée par les esprits protecteurs du clan, qui n'ont d'autre intérêt que la poursuite de la lutte et l'obtention de nouvelles victimes. Ainsi va grandir «Sênu le mêrgên nourri par les sêvên». Et, quand l'enfant devenu grand parviendra à la demeure de celui qui a tué son père et s'écriera : «Ô preux, toi qui as emmené ma mère et mon père, descends sur le rivage!", l'ennemi s'étonnera : «Je les avais pourtant tous tués!» (Sunik 1958, p. 115).

Celui qui a hérité le don chamanique de ses ancêtres est entraîné malgré lui dans la poursuite de la lutte qu'eux-mêmes ont menée autrefois : «Si tu ne te décides pas à combattre et si tu ne vaincs pas l'ennemi, explique la chamanesse Lindzja Bel'dy, ça ira mal, et tu mourras. Ce n'est pas drôle, c'est pénible. Quand on est jeune, on ne veut pas mourir, alors il faut se battre.»

Nikolaj Petrovič Bel'dy, au tout début de sa maladie chamanique, a été appelé à venger les chamanes de son clan qui avaient péri :

Mon grand-père, raconte-t-il, combattit avec un chamane étranger. Il fut vaincu et il mourut. J'avais donc dans l'autre monde un refuge spécial dëkaso où je pouvais revêtir ma cotte de mailles et ma cuirasse en fer, afin d'aller venger les chamanes de mon clan qui avaient péri avant moi. J'étais alors jeune et le sêvên qui devait m'accompagner dans mes périlleuses aventures pour m'aider dans mon combat ne m'était pas encore apparu. Mais ce sêvên avait déjà fait savoir à mon ennemi que je grandissais en futur vengeur. Et j'ai grandi, je suis devenu chamane! Le sêvên lui avait fait peur avec cette menace. Il lui avait fait peur longtemps avant. À lui, ou plutôt à l'un des siens, il en avait fait l'annonce en rêve, et les siens la lui avaient rapportée : «Tu as combattu, mais voilà que grandit celui qui se vengera de toi !»

Les chamanes qui s'affrontent dans ces duels ne se rencontrent pas en un combat réel. Ils continuent de vivre à des distances considérables l'un de l'autre. Comme le soutiennent les chamanes eux-mêmes, ce sont leurs âmes qui se rencontrent, ce qui expliquerait les longs sommeils de ces chamanes. Selon eux, la durée du duel du héros avec son adversaire décrite dans les contes est tout à fait réaliste et aucunement exagérée. Des jours et des nuits sans interruption, une année, trois années... Les chamanes dormiraient vraiment tout ce temps-là sans se réveiller. Et celui qui est condamné à la défaite serait le chamane qui, n'ayant su s'endormir à temps, est atteint par son ennemi alors qu'il est en état de veille, ou bien celui que l'on a réveillé en plein rêve du combat. À la mort de son mari, la tante de Kada Kile voulut se venger du chamane par la faute duquel il avait péri. Elle s'endormit et dormit sans se réveiller sept jours pleins.

Elle dormait nuit et jour, raconte Kada. J'ai pris peur. Je me suis approchée d'elle et je l'ai observée. Xêpurxêpur, elle respirait à peine. Elle avait dit: «Pendant que je dormirai, ne me touchez pas! » J'avais peur à la voir dormir nuit et jour. Non loin de nous, dans un coin du bois, il y avait un grand arbre. Le jour où ma tante devait se lever, cet arbre s'abattit à grand fracas, bien qu'il n'y eût ni vent ni quoi que ce soit. Une espèce de pierre semblable à une hache sans manche tomba du ciel, fendit l'arbre et s'y ficha. Alors seulement ma tante se leva en pleurant. «Tout est perdu, dit-elle, je me suis montrée moins bonne, plus faible que le chamane étranger.» 

Dans ce cas, il n'y a pas de description de duel, mais on nous raconte que le héros ${ }^{11}$ a dormi longtemps. «Eh bien, petite sœur [...] je dormirai neuf jours et neuf nuits. $\mathrm{Ne}$ laisse pas les enfants courir sur la berge!» (Avrorin 1986, p. 102). "“Petite sœur, je dormirai sept jours et sept nuits. Ne laisse pas les chiens aboyer ni les enfants courir sur la berge !" Et elle dormit sept jours pleins» (ibid. p. 134). On peut supposer que d'autres peuples que les Nanaïs croyaient eux aussi en des combats se déroulant en rêve. S'il en est ainsi, la conjonction des représentations du combat tel qu'il est rêvé par le rêveur (point de vue interne) et du long sommeil [tel qu'il est observé par le témoin] (point de vue externe) a pu donner naissance au motif du «sommeil ininterrompu de plusieurs jours qui saisit le preux avant le combat décisif» - motif largement répandu dans les récits épiques des peuples turcs et dans le répertoire international des contes merveilleux» (Žirmunskij 1974, p. 573).

L'épisode du duel entre le héros et son adversaire, malgré tous ses détails fantastiques, est accepté dans la tradition comme tout à fait réel. Les invraisemblances du duel avec des combattants qui s'entre-tuent, ressuscitent aussitôt et reprennent le combat s'expliqueraient selon les chamanes par le fait que ces luttes sont rêvées. D'autre part, toujours d'après eux, des phénomènes aussi étonnants se produiraient lors de combats réels (en état de veille). C'est que, chez le héros de conte comme chez le chamane, l'âme-force vitale (êrgên) se trouve dans un endroit inhabituel et inaccessible à l'ennemi. En effet, chez l'homme ordinaire, cette âme-force vitale est située dans le cœur ou dans la tête - endroits où un coup entraîne rapidement la mort. Une autre sorte d'âme (panjan), qui est le double ${ }^{12}$ de l'individu, peut quitter le corps à l'occasion d'un rêve ou d'une maladie: panjan et êrgên sont alors dissociées. Il n'en va pas de même pour le chamane. A. V. Smoljak la première a parlé d'une âme spécifique du chamane, le niokta (dans le dialecte nanaï du Sikhotê-Alin et en oultche : putê). D'après nos données, ce niokta réunirait en lui les esprits auxiliaires du chamane, son âme individuelle panjan et sa force vitale êrgên. Pendant les rêves ou les séances chamaniques, le niokta quitte le corps du chamane, emportant l'êrgên. Le chamane est alors invulnérable si l'on frappe son corps, mais terrassé par une mort soudaine et apparemment inexplicable si c'est son âme (qui se trouve alors loin de son corps) qui est frappée. Les Nanaïs pensent que les chamanes puissants cachent leur âme dans des endroits insolites ou difficiles d'accès pour être invulnérables ${ }^{13}$. Ainsi, selon les matériaux recueillis par P. P. Šimkevič, l'êrgên du chamane serait rangée dans une boite ou dans un œuf (Šimkevič 1986, p. 127). Dans les traditions notées par A. E. Gaer (1991, p. 86), les chamanes mettraient leur force vitale dans un quadrupède ou un oiseau, sur la pointe d'une aiguille, ou encore dans leur propre petit doigt. Ivan Torokovič Bel'dy se souvient d'un chamane du clan Posar que ses ennemis ne parvenaient pas à tuer. À la fin, épuisé par la multitude des blessures qu'il avait reçues, il leur dit: «Vous ne me tuerez jamais de la sorte. Voilà mon gros orteil. Coupez-le et je cesserai de vivre.»

Dans les contes nanaïs également, les deux protagonistes combattants sont invulnérables tant que leur êrgên reste dans son abri, loin du corps. La victoire n'échoit pas à celui qui a surpassé l'adversaire par la force, mais à celui qui a su le premier dénicher l'âme de son ennemi. Celle-ci est placée le plus souvent dans un œuf bizarre, 
noir, bicolore ou transparent et ressemble à un oisillon. Ainsi, dans le conte nanaï AT $302 / 1$, le héros provoque la mort de son ennemi en détruisant son âme grâce à ses esprits auxiliaires animaux - le succès de son entreprise venant de ce qu'il a su auparavant se concilier de ces esprits auxiliaires.

21 Tous ces motifs rencontrés dans le folklore des Nanaïs - la vengeance clanique, le sommeil du héros qu'il ne faut pas troubler et la recherche de l'âme de l'ennemi dans des objets extérieurs à son corps - se retrouvent dans le folklore de beaucoup d'autres peuples. Généralement, il n'est pas aisé de faire le lien entre ces motifs et les réalités ethnographiques sous-jacentes car «il y a eu une série de transformations progressives» (Putilov 1994, p.130). Les matériaux nanaïs, eux, conservent non seulement un stade archaïque de la tradition folklorique, mais également la vue du monde correspondant à ce stade; de ce fait, lesdites transformations sont accessibles au chercheur. Ainsi, notre étude des conceptions des chamanes nanaïs nous conduit à penser que les motifs relevés dans les nimman seraient le résultat de la transposition dans ces contes d'informations sur la lutte secrète et impitoyable que mènent les chamanes pour leur survie. En revanche, l'élucidation de la signification des motifs analogues rencontrés dans d'autres folklores exige à l'évidence une étude complète des transformations qui se sont produites au fur et à mesure que le contenu des contes passait de la réalité à la fiction.

\section{BIBLIOGRAPHIE}

Avrorin, V. A.

1986 Materialy po nanajskomu jazyku i fol'kloru (Leningrad).

Gaer, E. A.

1991 Tradicionnaja bytovaja obrjadnost' nanajcev v konce XIX-načale XX veka (Moscou).

Lopatin, I. A.

1922 Gol'dy amurskie, ussurijskie i sungarijskie (Vladivostok).

Meletinskij, E. M.

1963 Proisxoždenie geroičeskogo êposa. Rannie formy i arxaičeskie pamjatniki (Moscou).

Propp, V. Ja.

1986 Istoričeskie korni volšebnoj skazki (Leningrad).

Putilov, B. N.

1994 Fol'klor i narodnaja kul'tura (Saint-Pétersbourg).

Sem, Ju. A.

1986 Žanrovaja klassifikacija fol'klora nanajcev, Fol'klor i êtnografija narodov Severa (Leningrad).

Šimkevič, P. P.

1896 Materialy dlja issledovanija šamanstva u gol'dov (Khabarovsk).

Smoljak, A. V.

1991 Šaman : Ličnost', funkcii, mirovozzrenie, Narody Nižnego Amura (Moscou). 
Sunik, O.P.

1958 Kur-Urmijskij dialekt. Issledovanija i materialy po nanajskomu jazyku (Leningrad).

Žirmunskij, V. M.

1974 Tjurkskij geroičeskij êpos (Moscou).

\section{NOTES}

1. Le problème de la genèse du folklore a été abordé par B. N. Putilov (1994, p. 117).

2. Extrait des contes recueillis par l'auteur auprès de Nikolaj Petrovič Bel'dy et d'Ivan Torokovič Bel'dy dans les villages de Naïkhine (Najxin) et Daïerga (Daerga), territoire de Khabarovsk.

3. Traduction de l'auteur.

4. Le caractère chamanique des procédés mis en œuvre par le héros combattant du nigman nanaï a déjà été noté (Meletinskij 1963, p. 301).

5. Ces chants récitatifs ne se rencontrent pas dans d'autres types de récits ou d'invocations.

6. On peut réciter le conte dans la solitude avec les esprits pour seuls interlocuteurs.

7. [C'est-à-dire en état de veille. N.d.T.]

8. C'est-à-dire mon sêvên.

9. Traductions de conversations de l'auteur avec des chamanes nanaïs enregistrées au magnétophone.

10. [Généralement sous un chaudron renversé. N.d.T.]

11. [Qui est ici une héroïne. N.d.T.]

12. [L'ombre. N.d.T.]

13. Dans le même souci de protection, le chamane mettra les âmes des gens ordinaires dans des abris d'âmes collectifs dëkaso.

\section{RÉSUMÉS}

L'auteur interroge des chamanes nanaïs actuels sur la signification des contes épiques nanaïs, les ninman. D'après ces chamanes, la vengeance de sang décrite dans ces ninman n'est pas celle qu'ont pratiquée autrefois les Nanaïs dans leurs guerres claniques, mais celle que les lignées de chamanes entretiennent entre elles. Les combats à caractère fantastique relatés dans ces contes sont ceux-là mêmes que se livrent habituellement les chamanes rivaux (ou plutôt leurs âmes), durant leur sommeil, avec le concours de leurs esprits auxiliaires sêvên.

The author interviews present-day Nanai shamans on the meanings of the Nanai epic tales, the nimman. According to the shamans, the blood vengeance described in the nimmanis not that practiced in the past by the Nanais in their wars between clans, but rather the blood vengeance of the shamanic lineages. The fantastic battles recounted in these tales are the same as those usually held-in their sleep-by rival shamans (or rather by their souls) with the aid of their helping spirits, the sêvên. 
INDEX

Thèmes : chamanisme, littérature, représentations

Index géographique : Amour

nomsmotscles Nanai

Mots-clés : âme, contes, rêve, vengeance

\section{AUTEUR}

\section{TATIANA BULGAKOVA}

Tatiana Bulgakova est chercheur à l'Institut Pédagogique Hertzen à Saint-Petersburg. Anthropologue, elle a publié de nombreux articles sur le chamanisme, les pratiques rituelles et la littérature orale des Nanais (autrefois appelés Goldes), peuple toungouse de la Région de l'Amour, ainsi que sur les changements religieux contemporains chez les peuples tounguso-mandchous. 